\title{
Sector externo, complejidad y crecimiento económico desde la perspectiva latinoamericana
} External Sector, Complexity and Economic Growth in Latin America

\author{
Carlos A. Carrasco \\ Escuela de Negocios de la Universidad de Monterrey (UDEM) \\ carlos.carrasco@udem.edu
}


En los últimos cuarenta años, las economías latinoamericanas han sido partícipes del viraje mundial hacia la profundización de las relaciones económicas internacionales; no obstante, dicho cambio no se ha reflejado en un crecimiento económico sostenido que permita mejorar significativamente el nivel de vida de la región. Este artículo pretende servir de guía para futuras y más profundas discusiones sobre el tipo de política económica que hace posible el crecimiento económico sostenido y sostenible en la región latinoamericana. Para ello, se señalan las características del sector externo que se asocian con mayores tasas de crecimiento económico y se enfatizan los recientes desarrollos sobre complejidad económica que permiten identificar las industrias con mayor potencial para cada región. Posteriormente, se analiza gráficamente la relación entre estructura del sector externo, complejidad y crecimiento económico para un grupo de economías de la región y, finalmente, se discuten algunas implicaciones de política económica.

Palabras clave: sector externo, complejidad económica, crecimiento económico, cambio estructural, política industrial

In the last forty years, Latin American economies have participated in the global shift towards the deepening of international economic relations; however, this change has not been reflected in sustained rates of economic growth. This short article aims to encourage future and deeper discussions on the type of economic policy focused on the external sector that makes sustained and sustainable economic growth possible in the Latin American region. To this end, the article highlights the characteristics of the external sector that are associated with higher rates of economic growth and points out to the recent developments in economic complexity which allow identifying the industries with the greatest potential for each region. Thereafter, the relationship between external sector structure, complexity and economic growth for a group of economies in the region is analyzed and some economic policy implications are discussed.

Keywords: external sector, economic complexity, economic growth, structural change, industrial policy 


\section{Introducción}

El estudio del crecimiento económico tiene una larga tradición en la ciencia económica, tanto desde una perspectiva teórica como dentro de las prioridades de la política económica. Así, se han resaltado dos características deseables cuando se sitúa al crecimiento económico como objetivo prioritario de la política económica. Primero, el crecimiento económico debe ser sostenido en el tiempo, lo que se asocia con una mejora en la calidad de vida de las personas y del bienestar social. Segundo, el crecimiento económico debe ser sostenible de forma que considere los efectos sobre las generaciones futuras, el uso de recursos no renovables y las consecuencias en el medio ambiente. Además de lo anterior, un creciente número de estudios han señalado la necesidad de incorporar una dimensión distributiva dentro del estudio del crecimiento económico (Akinci, 2018; Arestis \& Troncoso, 2017; Stiglitz, 2016) que, sumado a lo anterior, permita mejorar de forma generalizada las condiciones de vida de la población.

La literatura económica ha identificado diversos determinantes del crecimiento económico como la dotación de factores, la acumulación de capital físico y humano, el cambio tecnológico, la estructura del sector externo y el marco institucional (Barro \& SalaiMartin, 2004; Carrasco \& Tovar-García, 2020b; Grossman \& Helpman, 1991; Panizza \& Presbitero, 2013; Wacziarg \& Welch, 2008). Incrementar la tasa de crecimiento económico ha estado entre los principales objetivos de política económica a nivel mundial; no obstante, en la mayor parte de los países en desarrollo, las tasas de crecimiento han estado lejos de los objetivos planteados, y esto incluye el caso de los países latinoamericanos. Más aún, en gran parte de Latinoamérica, el potencial de crecimiento de largo plazo se ha visto mermado ante la volatilidad de la actividad económica y la inestabilidad política, limitando la mejora en los estándares de vida de los habitantes de la región.

Estudios recientes se han enfocado en examinar las características del sector externo que permiten mejorar el desempeño económico e incrementar las tasas de crecimiento. Por ejemplo, se ha resaltado la importancia de la composición y diversificación de las exportaciones como determinantes tanto del balance externo como del crecimiento económico (Aditya \& Acharyya, 2013; Agosin, 2008; Carrasco \& Tovar-García, 2020a; TovarGarcía \& Carrasco, 2019), siendo especialmente relevantes las exportaciones de bienes de valor agregado alto. Además, se ha mostrado la relevancia de las importaciones de bienes de capital, lo que permite la acumulación de capital físico y la expansión de las capacidades productivas (Carrasco \& Tovar-García, 2020b; Herrerias \& Orts, 2013).

Desde el inicio de la crisis de la deuda de América Latina en los años ochenta, una parte de las economías latinoamericanas reestructuró su sector externo, diversificando la producción e incrementando el valor agregado de los bienes de exportaciones. No obstante, otro grupo de países de la región sigue dependiendo de las exportaciones de bienes básicos y materias primas, lo que limita los efectos derrame de las exportaciones e incrementa la vulnerabilidad a perturbaciones externas ante la volatilidad de los precios de dichos bienes en los mercados internacionales.

Transitar de exportaciones de bajo valor agregado a exportaciones de valor agregado medio y alto requiere de una modificación en la estructura productiva. En este sentido, los estudios sobre complejidad económica (Balland et al., 2020; Hidalgo, 2021; Hidalgo et al., 2018; Jun et al., 2020) pueden servir de guía en el camino a la reestructuración 
del sector externo. La complejidad económica implica la existencia de dependencia del pasado, interdependencia entre agentes y la evolución de las relaciones; por tanto, su estudio y uso en términos de política económica requiere de una visión amplia de las dinámicas económicas en diferentes niveles de agregación.

En este contexto, el objetivo de este artículo es doble. Por una parte, el artículo busca introducir la discusión actual en la literatura económica sobre la relación existente entre la estructura del sector externo, la complejidad del aparato productivo y el crecimiento económico. En segundo lugar, el artículo presenta un análisis exploratorio de dicha relación en el caso de las economías latinoamericanas. Una pregunta motiva la discusión que se plantea en este artículo: ¿cómo se puede transformar el sector externo de las economías latinoamericanas para que potencie el crecimiento económico sostenido y sostenible? Para lo anterior, se sigue una metodología exploratoria-descriptiva que permita una primera aproximación a la relación sector externo-complejidad económica-crecimiento para el caso de América Latina a partir de la revisión de la literatura y el análisis correlacional de datos del periodo 1980-2019.

Además de esta introducción, el artículo se estructura de la siguiente manera: la sección 2 hace una breve revisión de la literatura reciente que conecta la estructura del sector externo, la complejidad y el crecimiento económicos. En la sección 3 se presenta un análisis descriptivo de la situación actual del sector externo y la estructura económica de las economías latinoamericanas. En la sección 4 se discuten opciones de política económica que permitan mejorar las condiciones de crecimiento económico ligado a la estructura del sector externo. Finalmente, en la sección 5 se presentan las conclusiones.

\section{Sector externo, complejidad económica y política industrial}

Durante las décadas de los años ochenta y noventa, diversos países de Latinoamérica cambiaron su estrategia de desarrollo pasando del mercado interno como motor de crecimiento a una estrategia basada en la demanda externa a partir del impulso a las exportaciones (Wacziarg \& Welch, 2008). Algunos países reorganizaron el sector externo enfocándose en la producción de bienes manufacturados de intensidad tecnológica media y alta, mientras que otros mantuvieron al sector primario como eje de las industrias de exportación.

Existe una amplia literatura examinando la relación entre sector externo y crecimiento. Así, una parte de la literatura económica ha puesto énfasis en la debilidad de las estrategias de crecimiento basadas en las exportaciones — como lo sintetiza Palley (2012) _ , a la par que otra se ha enfocado en resaltar las características del sector externo que potencian el crecimiento económico.

En relación con el último punto, se ha subrayado que la composición de las exportaciones afecta al desempeño económico. Las exportaciones se asocian con mayor crecimiento económico cuando se diversifica de la exportación de materias primas y bienes básicos a la exportación de bienes manufacturados (Vogiatzoglou, 2019), aunque a partir de cierto umbral de desarrollo existen ganancias por la especialización (Munir \& Javed, 2018). Además, la relación se potencia cuando las empresas combinan un sesgo exportador con gasto en investigación y desarrollo (Bravo-Ortega et al., 2014). Más aún, la relación de las exportaciones con la acumulación de capital humano es clave: las exportaciones de manufacturas de valor agregado medio alto y alto requieren superar un umbral de acumulación 
de capital humano (Sheridan, 2014), a la vez que generan incentivos para la acumulación de capital humano (Blanchard \& Olney, 2017).

Por su parte, la relación entre el desempeño económico y las importaciones depende de diversos factores. Existen ganancias de la importación de bienes de capital al hacer posible la acumulación de capital y la expansión de la capacidad productiva (Carrasco \& Tovar-García, 2020b; Herrerias \& Orts, 2013); sin embargo, el crecimiento se ve mermado cuando las exportaciones incluyen una proporción alta de bienes importados (Carrasco \& Tovar-García, 2020b). Ahora bien, los efectos en el crecimiento económico de la importación de insumos dependen de las características de estos (Pierola et al., 2018) y de la parte del proceso de producción en donde se encuentren las industrias locales de exportación (Hagemejer, 2018).

En años recientes, se ha resaltado la relación entre la complejidad del sistema productivo, el sector externo y el crecimiento económico, pero antes de entrar en ello, es necesario definir qué es un sistema complejo. Un sistema complejo es aquel proceso evolutivo que presenta cierto nivel de orden como resultado de la interacción de diversos objetos heterogéneos, lo que permite entender fenómenos económicos al ilustrar cómo la interacción entre estructuras microeconómicas lleva a fenómenos económicos agregados (Durlauf, 1998). Por tanto, la incidencia en un sistema complejo requiere tomar en cuenta la interdependencia entre los agentes, la dependencia del pasado y la evolución constante de las relaciones.

La idea de complejidad económica ha ido ganando atención en el análisis del comercio internacional, la estructura productiva y el alcance potencial de la política industrial. Un concepto clave es el de parentesco (relatedness), que hace referencia a la medición de la afinidad total entre una actividad específica y un lugar o región, lo que permite entender las dependencias con el pasado y predecir el comportamiento de una actividad en dicho lugar (Hidalgo, 2021; Hidalgo et al., 2018). A lo anterior es necesario añadir los avances recientes en términos de medición de la complejidad económica a partir del desarrollo e implementación de técnicas de reducción de dimensiones (Hidalgo, 2021) que permiten analizar una gran cantidad de variables para cada región o localidad.

Teniendo en cuenta la anterior, la pregunta clave es: ¿cómo se puede impulsar la relación entre la estructura del sector externo, la complejidad económica y el crecimiento? La respuesta se encuentra en el uso de la política industrial. Por una parte, la literatura económica ha identificado las características del sector externo que hacen propicio el crecimiento económico. Por otro lado, la literatura sobre complejidad económica hace posible identificar qué producto o industria tiene posibilidades de desarrollo para cada región; o, por el contrario, dadas las características de la región, qué producto o industria es poco probable que se desarrolle con éxito. Con ello, es posible poner en marcha políticas industriales que estimulen el desarrollo de las industrias adecuadas para cada lugar y faciliten el flujo de conocimiento entre industrias y regiones (Hidalgo et al., 2018).

\section{La transformación del sector externo en Latinoamérica}

Las economías latinoamericanas han experimentado —en diferentes grados de intensidaduna transformación en su estructura económica como consecuencia del proceso de 
estabilización posterior a la crisis de deuda de los años ochenta y a las hiperinflaciones que se presentaron en algunos países de la región entre finales de dicha década y principios de los años noventa. Dentro de las principales reformas se encuentra la transformación del sector externo a partir de una mayor apertura comercial y de la disminución o eliminación de las barreras de entrada a la inversión extranjera directa (IED) (Williamson, 2004).

El proceso de reformas del sector externo se ha manifestado de distintas maneras y niveles: en un principio, con la apertura comercial unilateral y, posteriormente, con las firmas de acuerdos comerciales y la puesta en marcha de mecanismos de integración económica más profundos, como es el caso del Mercado Común del Sur (Mercosur). Esta transformación se ha reflejado en un incremento de la apertura comercial, medida como la suma de exportaciones e importaciones como proporción del producto interno bruto (PIB).

Para ejemplificar la transformación del sector externo y los retos que enfrenta la región, a continuación se retoman datos de los diez países latinoamericanos más poblados'. En cuanto a la apertura comercial, si comparamos 1980 (antes del proceso de apertura) y 2019 (año más reciente para el que hay datos) se aprecia que en Argentina esta pasó de 11,5 al 32,6 \%, en Bolivia del 46,8 al 56,4 \%, en Brasil del 20,3 al $29 \%$, en Chile del 48,1 al 56,8 \%, en Colombia del 31,8 al 37,7 \%, en Ecuador del 35 al 46,7 \% y en México del 22,4 al 77,9 \%. De los diez países más poblados de la región, únicamente tres presentan una menor o igual apertura comercial: Guatemala, que pasó de 47,1 al 45,9 \%; Perú, que tuvo una variación mínima de 47,6 al 47,12 \%; y Venezuela, que cambió de 57,4 al 48,1 \% (en 2014, último dato disponible). Si bien los datos de apertura comercial son susceptibles a los precios de los bienes de importación y exportación, y a la moneda que se utiliza como referencia para hacer las estimaciones, una visión agregada permite afirmar que la región es ahora más abierta al comercio exterior de lo que fue en $1980^{2}$.

Aunado a la mayor apertura comercial, la composición de las exportaciones también ha cambiado, como se puede observar en la figura 1. En este sentido, hay dos factores que ayudan a explicar el cambio. Por una parte, la estrategia de apertura de algunos países de la región priorizo la transformación del sector externo, incrementando la participación de bienes manufacturados de valor agregado medio y alto como parte de la incorporación de estos países en las cadenas globales de valor. En este grupo de países se encuentran, principalmente, México y Guatemala; no obstante, también se podría incluir a Brasil y Colombia. Por otra parte, durante la primera década del siglo XXI, el precio de las materias primas y de los bienes básicos y energéticos se incrementó sustancialmente, lo que acrecentó la participación de este tipo de bienes en las exportaciones de mercancías. Esto explica el incremento sustancial en la participación de las exportaciones de combustibles, materias primas agrícolas, comida, y metales y minerales en Argentina, Bolivia, Colombia, Ecuador, Perú y Venezuela.

\footnotetext{
1 Los datos que se presentan en esta sección corresponden al periodo 1980-2019 para los diez países más poblados de América Latina: Argentina, Bolivia, Brasil, Chile, Colombia, Ecuador, Guatemala, México, Perú y Venezuela.

2 Los máximos de apertura durante el periodo 1980-2019 difieren sustancialmente entre países: 41,7 \% (2002) en Argentina, 85,3 \% en Bolivia (2014), 29,7 \% en Brasil (2004), 80,8 \% en Chile (2008), 39,6 \% en Colombia (2006), 68 \% en Ecuador (2008), 69,5 \% en Guatemala (2001), 80,6 \% en México (2018), 58,4 \% en Perú (2008) y $60,1 \%$ en Venezuela (2005).
} 


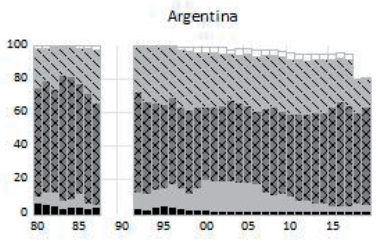

Chile

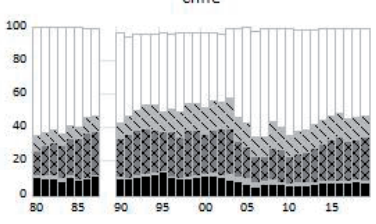

Guatemala

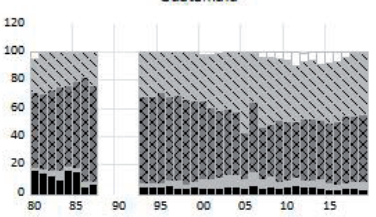

Venezuela

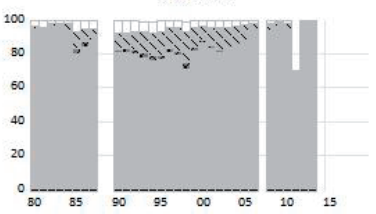

Bolivia
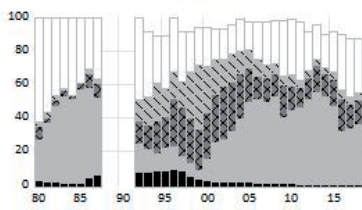

Colombia

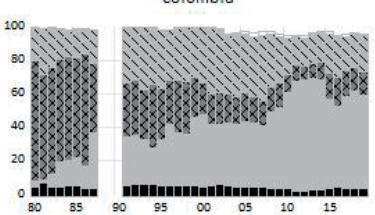

México

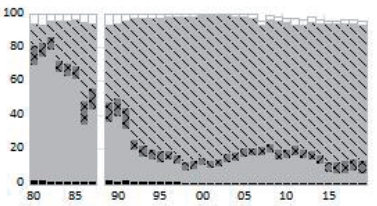

Brasil

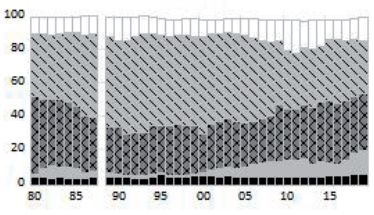

Ecuador
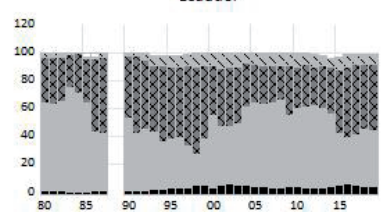

Perí

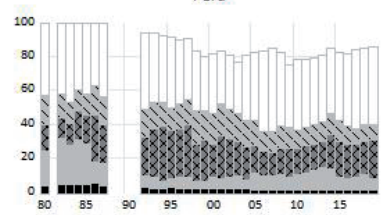

Fuente: elaboración propia con datos de los indicadores mundiales de desarrollo del Banco Mundial.

Como se desarrolla en la sección anterior, la literatura económica ha analizado la relación entre la estructura del sector externo y el crecimiento económico. ¿Qué dicen los datos para el caso latinoamericano? Si bien este artículo es exploratorio, la figura 2 ofrece indicios de la relación existente entre el crecimiento económico, la estructura del sector externo y la complejidad económica. La figura 2.A muestra la relación entre el PIB per cápita y la apertura económica. Como se observa gráficamente, la relación no es clara. La apertura comercial no se explica únicamente por decisiones de política económica, sino que depende de elementos tales como el tamaño del país y las dotaciones de factores productivos.

La figura 2.B muestra la relación entre PIB per cápita y las exportaciones de alta tecnología. En este caso, la gráfica parece mostrar una relación positiva. La literatura económica ha señalado la importancia de las exportaciones de alta tecnología en el desempeño económico y el balance con el exterior. No obstante, dicha relación depende parcialmente del contenido importado de las exportaciones; es decir, a mayor contenido importado de las exportaciones, menores efectos tendrán estas en el crecimiento. En cuanto a la conexión entre PIB per cápita y diversificación de las exportaciones (figura 2.C), la relación no es clara. La literatura económica ha señalado la existencia de una relación no lineal. Así, se aprecia que la transición de exportaciones de materias primas y bienes básicos a manufacturas de valor agregado medio y alto promueve el crecimiento económico; sin embargo, a partir de cierto umbral de desarrollo, los países obtienen mayores ganancias a partir de la especialización en aquellos bienes en los que desarrollan ventajas comparativas. 
La figura 2.D muestra la relación positiva entre el PIB per cápita y el índice de complejidad económica3; en otras palabras, una mayor complejidad económica se asocia con un nivel más alto del PIB per cápita. Ahora bien, es posible conectar la estructura del sector externo con la complejidad de la estructura productiva y el nivel de PIB per cápita, como se observa en la figura 2.E, que muestra la relación entre el índice de complejidad económica y el índice de diversificación de las exportaciones. En este caso, aunque existen indicios de una relación negativa, el gráfico no es claro. Lo anterior se relaciona con la no linealidad entre la diversidad de las exportaciones y el desempeño económico que ya se ha señalado previamente. Finalmente, la figura 2.F muestra una relación positiva entre exportaciones de alta tecnología, caracterizadas por un valor añadido alto, y la complejidad de la estructura económica.

Si bien el presente es un artículo exploratorio, su motivación radica en señalar el camino tanto para profundizar el estudio de la relación entre la estructura del sector externo, la complejidad y el crecimiento económico; como para delinear la vía a explorar por parte de los hacedores de política económica, de manera que se potencie dicha relación y se logre alcanzar el objetivo de un crecimiento económico sostenido y sostenible.

Figura 2. Sector externo, complejidad y crecimiento económico

A

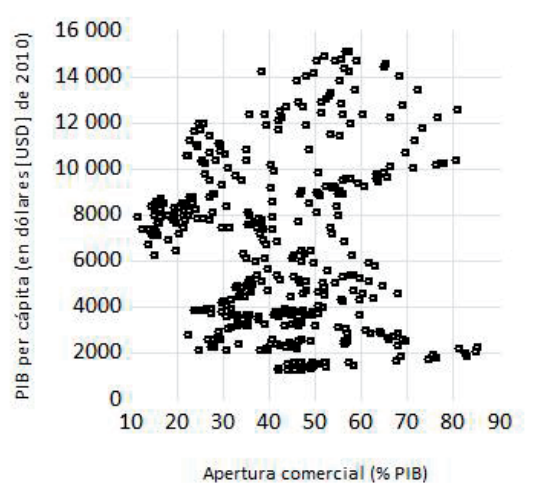

C

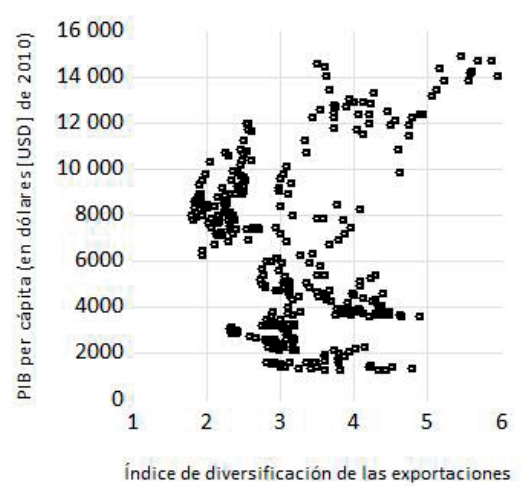

B

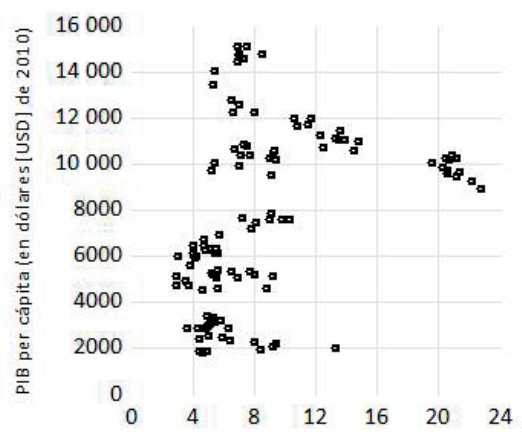

Exportaciones de alta tecnología (\% exportaciones de manufacturas)

D

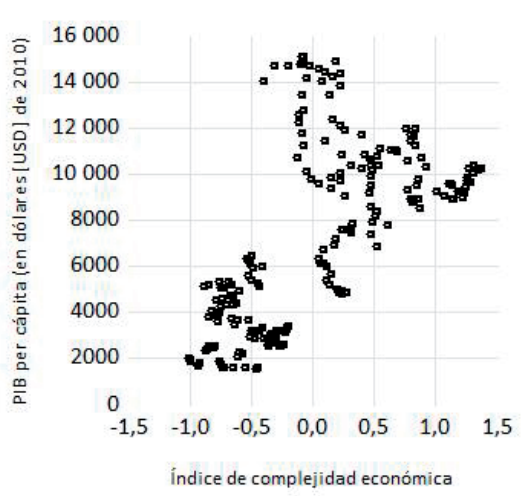

3 El índice de complejidad económica se incrementa cuando un país o región incrementa el número y complejidad de los productos que exporta. 

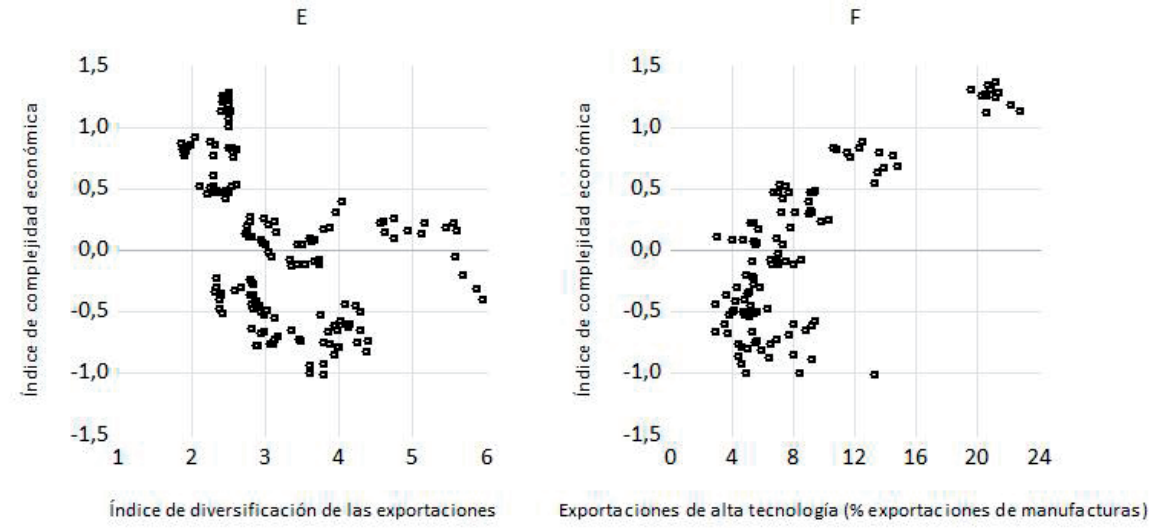

Fuente: elaboración propia con datos de los indicadores mundiales de desarrollo del Fondo Monetario Internacional y de Simoes e Hidalgo (2011).

\section{Política económica y la trayectoria del sector externo}

Recientemente, la economía mundial ha observado un cambio estructural hacia la relocalización de los procesos productivos a partir de la profundización de las relaciones económicas internacionales dentro de las cadenas globales de valor. Con una creciente competencia de los países en desarrollo y emergentes, la actividad manufacturera de los países desarrollados ha perdido peso relativo a nivel global. No obstante, este cambio se ha visto acompañado de una mayor demanda por manufactura eficiente, mayor dinamismo y complejidad en los procesos productivos, un acelerado cambio tecnológico en el marco de la cuarta revolución industrial y la participación de países de diferentes partes del mundo en las cadenas globales de valor (O'Sullivan et al., 2013).

En este contexto, si el objetivo es una transformación de la estructura productiva que facilite el crecimiento económico, la puesta en marcha de una nueva estrategia de política industrial debe ser prioritaria. La política industrial permite estimular actividades económicas específicas, haciendo posible el cambio estructural (Rodrik, 2009) al tiempo que lidia con las interdependencias estructurales, la construcción de instituciones y el manejo del conflicto (Andreoni \& Chang, 2019).

¿Qué camino se requiere seguir para impulsar el cambio en la estructura económica de los países latinoamericanos? La pregunta es ambiciosa y de difícil respuesta. A pesar de ello, es posible delinear algunos puntos que se deben contemplar en el marco de la implementación de una política industrial que potencie la relación entre sector externo, complejidad y crecimiento. Desde el inicio, es necesario señalar que la política industrial debe tener una visión de largo plazo y, a la vez, la flexibilidad de adaptarse al cambio. En el marco de un sistema complejo, la política industrial debe contemplar que el cambio en la estructura productiva implica un proceso evolutivo con dependencia del pasado, interdependencia entre actores y un presente que influye en el futuro. Además, las características propias del país y la región importan en la implementación de medidas de política industrial y, por tanto, el aterrizaje y selección específica de las políticas económicas, así como su implementación, requieren de una discusión a nivel local. 
Una pregunta clave es qué industrias potenciar, teniendo siempre en mente un horizonte de largo plazo. La respuesta a esta pregunta parece encontrar cierto consenso. La urgencia ambiental nos hace enfocar la transición hacía las energías limpias y una estructura económica ambientalmente amigable como prioridad dentro de la estrategia de desarrollo y como eje central de la política industrial (Åhman \& Nilsson, 2015; Meckling \& Allan, 2020; Pollin, 2021). No obstante, también cabe subrayar que los recientes desarrollos sobre complejidad económica permiten identificar a nivel local las industrias o áreas con un mayor potencial de desarrollo.

La apuesta hacia actividades que prioricen la transición energética y aquellas intensivas en investigación y desarrollo requiere de la coordinación entre el sector privado, el sector público y las universidades (Lerman et al., 2021) en un marco de constante evolución. Así, una clave para el éxito del cambio del sector externo es la acumulación de capital humano (Sheridan, 2014; Blanchard \& Olney, 2017) a través del acceso generalizado a servicios de salud y educación de calidad.

\section{Conclusiones}

El camino al desarrollo económico es largo y sinuoso. Aunque una parte importante de la literatura económica se ha centrado en el análisis de las variables que hacen posible el crecimiento económico, la puesta en marcha de políticas económicas que permiten a una economía crecer no ha sido del todo exitosa. El rezago relativo de la región latinoamericana tiene costos en términos de crecimiento económico y, con ello, influye en el bienestar de los habitantes de la región.

Durante la reconfiguración de la estrategia de desarrollo posterior a las crisis de deuda y balanza de pagos de los años ochenta, la política industrial sufrió un rechazo generalizado (Andreoni \& Chang, 2019). Sin embargo, la conexión existente entre el crecimiento económico, el sector externo y la complejidad del aparato productivo hacen urgente una reconsideración de las estrategias de desarrollo industrial.

La estrategia de desarrollo en cada país de la región debe contemplar los constantes cambios en la economía mundial. En este sentido, la implementación de políticas industriales enfocadas en la transformación del aparato productivo deberá tener una visión de largo plazo, así como un grado de flexibilidad suficiente que permita su modificación ante cambios coyunturales. Dichas políticas deberán contemplar las características propias de cada país y región, incrementando así las probabilidades de éxito de la estrategia de transformación.

Finalmente, es necesario destacar que el presente trabajo es una primera aproximación que busca motivar a más investigadores a profundizar en la relación entre sector externo, complejidad y crecimiento para el caso latinoamericano. Por ello, las limitantes del trabajo deberán ser abordadas en investigaciones futuras que permitan mejorar la toma de decisiones de política pública conducentes a la transformación del sector externo. 


\section{bibliografía}

Aditya, A., \& Acharyya, R.

2013 Export diversification, composition, and economic growth: Evidence from cross-country analysis. The Journal of International Trade \& Economic Development, 22(7), 959-992. https://doi.or g/10.1080/09638199.2011.619009

\section{Agosin, M. R.}

Export diversification and growth in emerging economies. CEPAL Review, 97, 115-131.

\section{Åhman, M., \& Nilsson, L. J.}

Decarbonizing Industry in the EU: Climate,

Trade and Industrial Policy Strategies.

En C. Dupont y S. Oberthür (eds.),

Decarbonization in the European Union

(pp. 92-114). Palgrave Macmillan UK.

https://doi.org/10.1057/9781137406835_5

\section{Akinci, $\mathbf{M}$.}

2018

Inequality and economic growth: Trickledown effect revisited. Development Policy Review, 36, 01-O24. https://doi. org/10.1111/dpr.12214

\section{Andreoni, A., \& Chang, H.-J.}

The political economy of industrial policy:

Structural interdependencies, policy alignment and conflict management. Structural Change and Economic Dynamics, 48, 136-150. https://doi. org/10.1016/j.strueco.2018.10.007

\section{Arestis, P., \& Troncoso, B.}

Balland, P.-A., Jara-Figueroa, C., Petralia, S. G., Steijn, M. P. A., Rigby, D. L., \& Hidalgo, C. A.

2020

Glob
investment: Export composition drives educational attainment. Journal of International Economics, 106, 165-183. https://doi.org/10.1016/j.jinteco.2017.03.004

\section{Bravo-Ortega, C., Benavente, J. M., \& González, Á.}

2014 Innovation, Exports, and Productivity: Learning and Self-Selection in Chile. Emerging Markets Finance and Trade, 50(sup1), 68-95. https://doi.org/10.2753/ REE1540-496X5001S105

\section{Carrasco, C. A., \& Tovar-García, E. D.}

2020a Export composition and Eurozone trade balance in manufacturing goods. Romanian Journal of Economic Forecasting, 23(1), 134-150.

\section{Carrasco, C. A., \& Tovar-García, E. D.}

2020b Trade and growth in developing countries: The role of export composition, import composition and export diversification. Economic Change and Restructuring, (54), 919-941. https://doi.org/10.1007/s10644020-09291-8 


\section{bibliografía}

Durlauf, S. N.

1998

What should policymakers know about economic complexity? The Washington Quarterly, 21(1), 155-165. https://doi. org/10.1080/01636609809550300

\section{Grossman, G. M., \& Helpman, E.}

1991 Trade, knowledge spillovers, and growth.

European Economic Review, 35(2-3),

517-526. https://doi.org/10.1016/0014-

2921(91)90153-A

\section{Hagemejer, $\mathbf{J}$.}

Trade and Growth in the New Member States: The Role of Global Value Chains. Emerging Markets Finance and Trade, 54(11), 2630-2649. https://doi.org/10.1080/1 540496X.2017.1369878

\section{Herrerias, M. J., \& Orts, V.}

Capital goods imports and long-run growth: Is the Chinese experience relevant to developing countries? Journal of Policy Modeling, 35(5), 781-797. https://doi. org/10.1016/j.jpolmod.2013.02.006

\section{Hidalgo, C. A.}

Economic complexity theory and applications. Nature Reviews Physics, 3(2), 92-113. https:// doi.org/10.1038/s42254-020-00275-1

Hidalgo, C. A., Balland, P.-A., Boschma, R., Delgado, M., Feldman, M., Frenken, K. Glaeser, E., He, C., Kogler, D. F., Morrison, A., Neffke, F., Rigby, D., Stern, S., Zheng, S., \& Zhu, S.
Morales, C. Gershenson, D. Braha,

A. A. Minai e Y. Bar-Yam (eds.), Unifying

Themes in Complex Systems IX

(pp. 451-457). Cham: Springer. https://doi.

org/10.1007/978-3-319-96661-8_46
Jun, B., Alshamsi, A., Gao, J., \& Hidalgo, C. A.

2020

Bilateral relatedness: knowledge diffusion and the evolution of bilateral trade. Journal of Evolutionary Economics, 30(2), 247-277. https://doi.org/10.1007/s00191-019-00638-7

\section{Lerman, L. V., Gerstlberger, W.,} Ferreira Lima, M., \& Frank, A. G.

How governments, universities, and companies contribute to renewable energy development? A municipal innovation policy perspective of the triple helix. Energy Research \& Social Science, 71, 101854. https://doi.org/10.1016/j. erss.2020.101854

\section{Meckling, J., \& Allan, B. B.}

The evolution of ideas in global climate policy. Nature Climate Change, 10(5), 434-438. https://doi.org/10.1038/s41558020-0739-7

\section{Munir, K., \& Javed, Z.}

2018 Export composition and economic growth:

Evidence from South Asian countries. South Asian Journal of Business Studies, 7(2), 225-240. https://doi.org/10.1108/ SAJBS-10-2017-0117

O'Sullivan, E., Andreoni, A., Lopez-Gomez, C., \& Gregory, M.

2013 What is new in the new industrial policy? A manufacturing systems perspective, Oxford Review of Economic Policy, 29(2), 432-462.

\section{Palley, T. I.}

The rise and fall of export-led growth. Investigación Económica, 71(280). https://doi.org/10.22201/ fe.01851667p.2012.280.37339 


\section{bibliografía}

Panizza, U., \& Presbitero, A. F.

2013

Public debt and economic growth in

advanced economies: A survey. Swiss

Journal of Economics and Statistics,

149(2), 175-204. https://doi.org/10.1007/

BF03399388

Pierola, M. D., Fernandes, A. M., \& Farole, $\mathrm{T}$.

2018

The role of imports for exporter

performance in Peru. The World Economy,

41(2), 550-572. https://doi.org/10.1111/

twec. 12524

\section{Pollin, R.}

2021 The industrial policy requirements for a global climate stabilization project.

International Review of Applied

Economics, 35(3-4), 389-406. https://doi.or

g/10.1080/02692171.2020.1755239

\section{Rodrik, D.}

Industrial Policy: Don't Ask Why, Ask

How. Middle East Development Journal,

1(1), 1-29. https://doi.org/10.1142/

S1793812009000024

\section{Sheridan, B. J.}

Manufacturing exports and growth: When is a developing country ready to transition from primary exports to manufacturing exports? Journal of Macroeconomics, 42, 1-13. https://doi.org/10.1016/j. jmacro.2014.06.002

\section{Simoes, A. J. G., \& Hidalgo, C. A.}

An Analytical Tool for Understanding the

Dynamics of Economic Development.

Workshops at the Twenty-Fifth AAAI

Conference on Artificial Intelligence.

\section{Stiglitz, J. E.}

2016

Inequality and Economic Growth. En

M. Jacobs y M. Mazzucato (eds.),

Rethinking Capitalism: Economics and

Policy for Sustainable and Inclusive

Growth (pp. 134-155). Wiley-Blackwell.

https://academiccommons.columbia.edu/

doi/10.7916/d8-dg8s-qk19/download

\section{Tovar-García, E. D., \& Carrasco,}

\section{A.}

2019

Export and import composition as

determinants of bilateral trade in goods: evidence from Russia. Post-Communist Economies, 31(4), 530-546. https://doi.org/ 10.1080/14631377.2018.1557913

\section{Vogiatzoglou, K.}

Export Composition and Long-Run

Economic Growth Impact: A Cointegration Analysis for ASEAN 'Latecomer'

Economies. Margin: The Journal of Applied Economic Research, 13(2), 168-191. https:// doi.org/10.1177/0973801018812571

\section{Wacziarg, R., \& Welch, K. H.}

Trade Liberalization and Growth: New

Evidence. The World Bank Economic Review, 22(2), 187-231. https://doi. org/10.1093/wber/lhn007

\section{Williamson, J.}

The strange history of the Washington consensus. Journal of Post Keynesian Economics, 27(2), 195-206.

Fecha de recepción: 1 de junio de 2021 Fecha de aprobación: 2 de octubre de 2021 Fecha de publicación: 21 de noviembre de 2021 